\title{
Investigation and Simulation Study on the Cascading Trip-off Fault of a Large Number of Wind Turbines in China on May 14, 2012
}

\author{
Ying Qiao*, Zong-Xiang Lu*, Ji Lu ${ }^{\dagger}$, Jia-Yang Ruan* and Lin-lin Wu**
}

\begin{abstract}
The integration of the large-scale wind power brings great challenge to the stability of the power grid. This paper investigates and studies the fault on May 14, 2012 of the large-scale cascading trip-off of wind turbines in North China. According to the characteristics of the voltage variation, the fault process is divided into three stages: the pre-event stage, the critical stage before cascading, and the cascading stage. The scenes in the fault are reproduced, using the full-size actual power system model. Simulation models of double-fed induction generators (DFIGs) and SVCs including protection settings and controller strategies are carefully chosen to find out the reason of voltage instability in each stage. Some voltage dynamic that have never been observed before in the faults of the same kind are analyzed in detail, and an equivalent voltage sensitive dynamic model of DFIG is proposed for the fast computation. The conclusions about the voltage dynamics are validated by the actual PMU observation evidence.
\end{abstract}

Keywords: Wind power, Fault analysis, Cascading trip-off, Voltage instability, Overvoltage

\section{Introduction}

As the most promising type of new clean energy, wind power has attracted the worldwide attention. Up to June, 2014, the worldwide wind capacity had reached $336 \mathrm{GW}$ [1]. With the increasing of wind power, its effects on system operation have become more and more prominent. In recent years, accidents that incurred trip-off of largescale wind turbines have been reported in countries [2]. Large-scale wind power bases of GW level have been constructed in China, and the wind power has been transmitted to the HV and EHV systems via long distance lines. These weak transmission systems are often threatened by voltage instability. It was reported that there had been 205 times of faults or failures in China from 2011 to 2012 [3].

Many research has contributes to the voltage stability of the large wind power system, such as the structural bifurcation analysis [4], the stability margin estimation [56], and the approaches to improve the voltage stability [78]. [9] analyzed the impact of wind power in the Jeju Island Power System. [3] studied the mechanism of the cascading trip-off of wind turbines and analyzes the process of the failure. [10] proposed a methodology based on a Z-bus algorithm to assess the tripping status of wind farms before the fault. Those researches were carried out in the simplified systems, and they could provide useful

$\dagger$ Corresponding Author: State Key Lab of Power Systems, Department of Electrical Engineering, Tsinghua University, Beijing 100084, China. (lujoin163@163.com)

* State Key Lab of Power Systems, Department of Electrical Engineering, Tsinghua University, Beijing 100084, China.

** North China Electric Power Research Institution, Beijing 100045, China.

Received: January 12, 2015; Accepted: July 28, 2015 information, but only some basic fault scenarios were modeled. The simulated results could hardly figure out the complicated situations of the actual system. Few papers have been found in the instability analysis of the large wind power whose simulation model could be validated by the measured data. [11] provided the comparison between the measured and simulated curves during the voltage dip, but the case was much simpler than what occurred in China.

In the fault analysis, it is essential to find out the inherence of the instability and identify which is the most dangerous risk factor. However, in the actual system, many protection and control strategies would be put into use once the system is suffering from serious disturbances. The dynamic process could be interrupted or changed. It is hardly observed that the measured waveform matched the simulated one. On the other hand, some unusual dynamic processes could be observed and they could not be explained by conventional models, so the new models must be proposed to reveal what really happened.

Based on the actual PMU records, this paper investigates the entire process of one cascading fault in North China on May 14, 2012. The scene of the fault is then elaborately reconstructed to analyze the reason of voltage instability. Some slow dynamic characteristics of the voltage were observed for the first time in the PMU records, and then its mechanism is simulated based on the proposed voltage sensitive model of DFIGs.

Section 2 introduces the grid configuration of the wind power base. Section 3 describes the fault evolution by dividing it into three stages: the pre-event stage, the critical stage and the cascading stage. Section 4 analyzes on the voltage stability of different stages qualitatively. Proper simulation models of multi-time scales are carefully chosen 
to reproduce the fault scene in Section 5, using the topology structure, parameters, protection settings and control strategies of the actual power grid. Conclusions are drawn in Section 6.

\section{The Structure Features of the Wind Power Base}

On May.14, 2012, a severe fault occurred in North China power grid, resulting in the cascading trip-off of 584 wind turbines in 15 wind farms with the loss of $737.1 \mathrm{MW}$ wind power in total. [12].

Fig. 1 shows the power grid before the fault, and the grid was the transmission system composing of the $500 \mathrm{kV}$-level subsystem and the $220 \mathrm{kV}$-level subsystem. 23 wind farms were connected to the $\mathrm{A}, \mathrm{B}, \mathrm{C}$, and $\mathrm{D}$ gathering substations via $220 \mathrm{kV}$ or $110 \mathrm{kV}$ bus, and then wind power was transmitted to North China Power Grid through two main transformers with the capacity of 750MVA. At that time, the wind power base had 1191 wind turbines with the rated capacity of $2133 \mathrm{MW}$ totally. The grid was the pure wind power integration system without any load or synchronous generator, and the total length of all transmission lines of the system was over $700 \mathrm{~km}$.

All 23 wind farms were equipped with dynamic

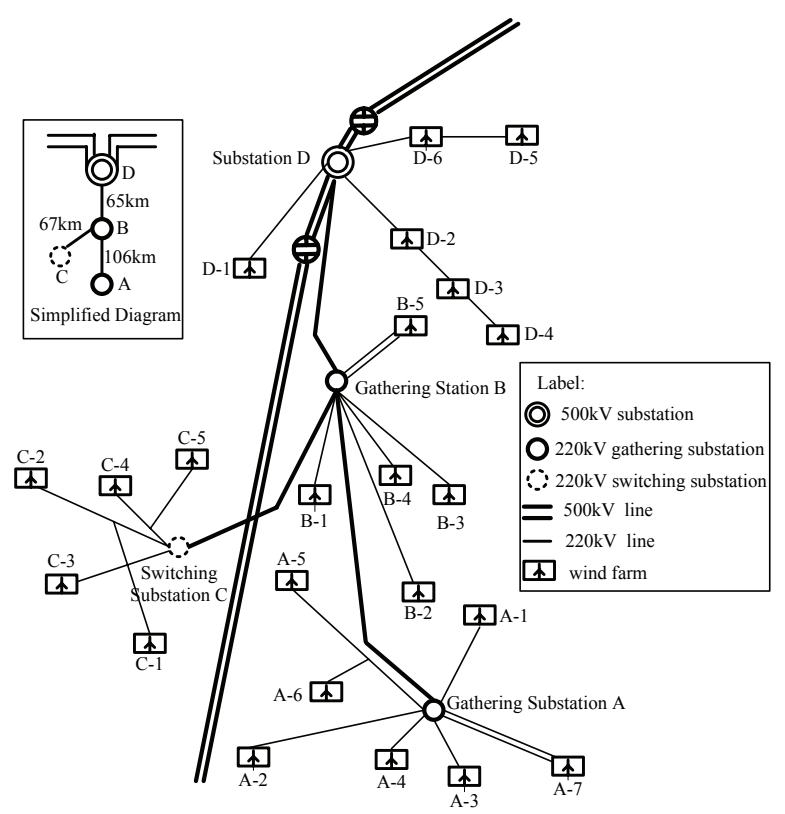

Fig. 1. The grid of the wind power base

Table 1. Reactive power compensation of gathering stations

\begin{tabular}{c|c|c|c|c}
\hline \multirow{2}{*}{$\begin{array}{c}\text { Gathering } \\
\text { Station }\end{array}$} & \multicolumn{2}{|c|}{ Capacitor group } & \multicolumn{2}{c}{ Reactor group } \\
\cline { 2 - 5 } & Group & $\begin{array}{c}\text { Capacity of } \\
\text { each bank }\end{array}$ & Group & $\begin{array}{c}\text { Capacity of } \\
\text { each group }\end{array}$ \\
\hline A & 8 & 10Mvar & 4 & $10 \mathrm{Mvar}$ \\
\hline B & 8 & $12 \mathrm{Mvar}$ & 4 & $12 \mathrm{Mvar}$ \\
\hline C & $/$ & $/$ & $/$ & $/$ \\
\hline D & 4 & 60Mvar & $/$ & $/$ \\
\hline
\end{tabular}

reactive compensation devices, static var compensators (SVCs), or fixed capacitors and reactors, which were connected with $35 \mathrm{kV}$ buses. Table 1 shows the reactive power compensation of all gathering substations.

\section{Investigation on the Cascading Trip-off Fault}

There were 4 turning points in the process of the fault:

(1) Since 13:20:00, the output power of all wind farms increased rapidly and then the load flow grew, which led to the reduction of the system voltage. In order to recovery the voltage level, both the gathering substations and the wind farms increased their capacitive compensation. The cut-off operation of reactors and the cut-in operation of capacitors resulted in drastic voltage fluctuation.

(2) At 13:41:16, a capacitor branch with the capacity of $23.76 \mathrm{Mvar}$ in the A-2 wind farm was put into operation, and then the system voltage kept increasing slowly.

(3) About 5.5 seconds later, at 13:41:21.5, the voltages of some points of common coupling (PCC) exceeded the threshold of the overvoltage protection of wind turbines, and subsequently a large number of wind turbines tripped off in the cascading mode. The loss of a large amount of real power finally incurred frequency decreasing.

(4) At 13:41:31, the reactive compensation devices gradually quitted due to overvoltage protection, and the system voltage restored to normal.

According to the four turning points, the total process of the fault is divided into four stages. The paper introduces the first three stages in detail.

\subsection{The Pre-event stage of the power system}

The stage was called the pre-event stage in the time range from 13:20:00 to 13:41:16. The output of wind power increased in general, although it declined for a very short time with the decreasing of the wind speed. Fig. 2 shows the active power flow from A-B and B-D, respectively.

At 13:31:00, the active power output increased more rapidly, resulting in more reactive consumption of transmission lines and the significant reduction of common coupling bus voltages (Fig. 3). The reactive power compensation devices of substations and multiple wind farms were put into operation to recover the voltage. The system voltage fluctuated with the repeating on/off operation of capacitors, as shown in Fig. 4.

\subsection{The critical stage before cascading}

As the system voltage was low, a capacitor branch of 


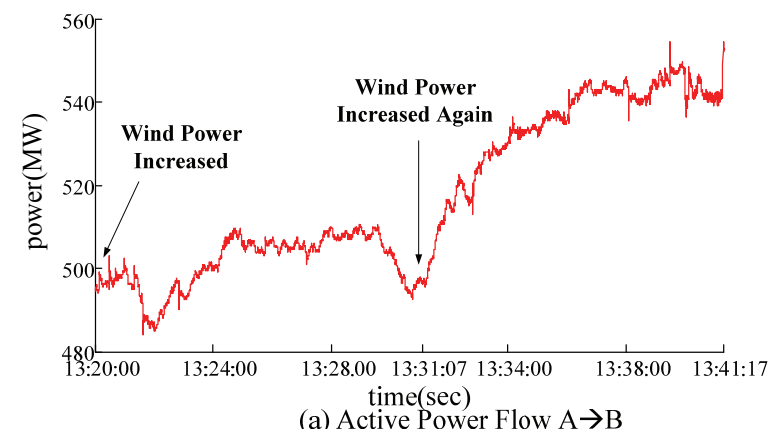

(a) Active Power Flow $\mathrm{A} \rightarrow \mathrm{B}$

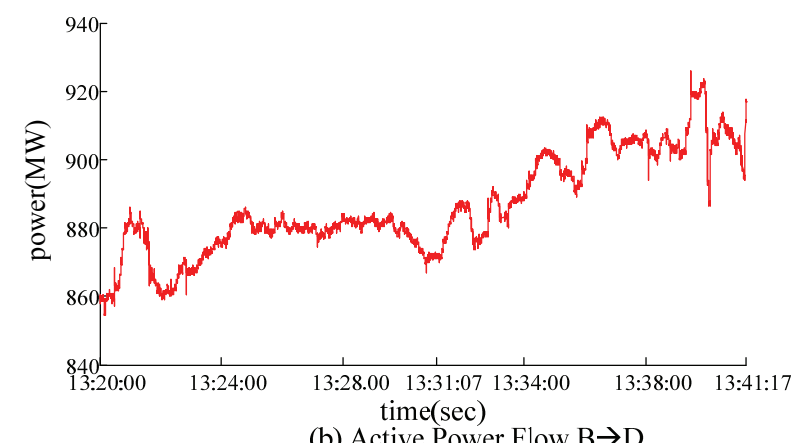

Fig. 2. The active power flow before cascading

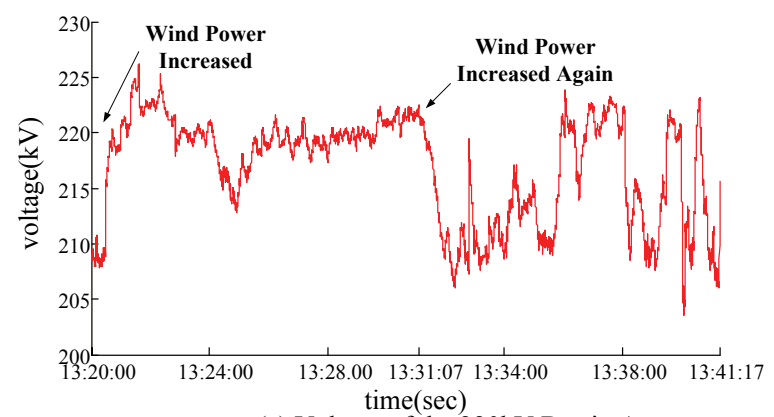

(a) Voltage of the $220 \mathrm{kV}$ Bus in $\mathrm{A}$

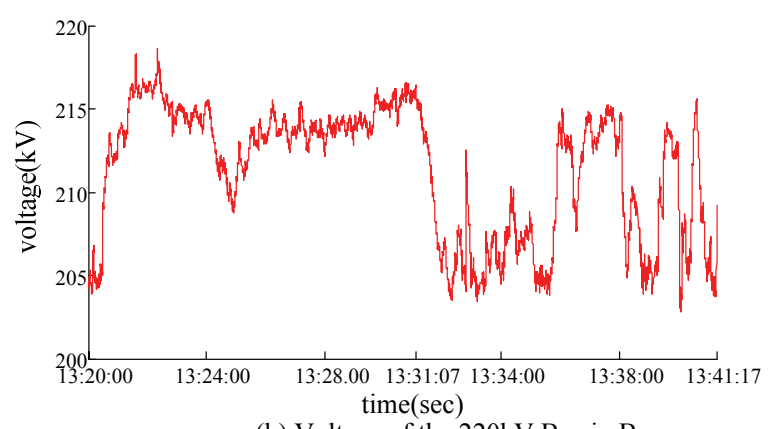

(b) Voltage of the $220 \mathrm{kV}$ Bus in B

Fig. 3. The voltage before cascading

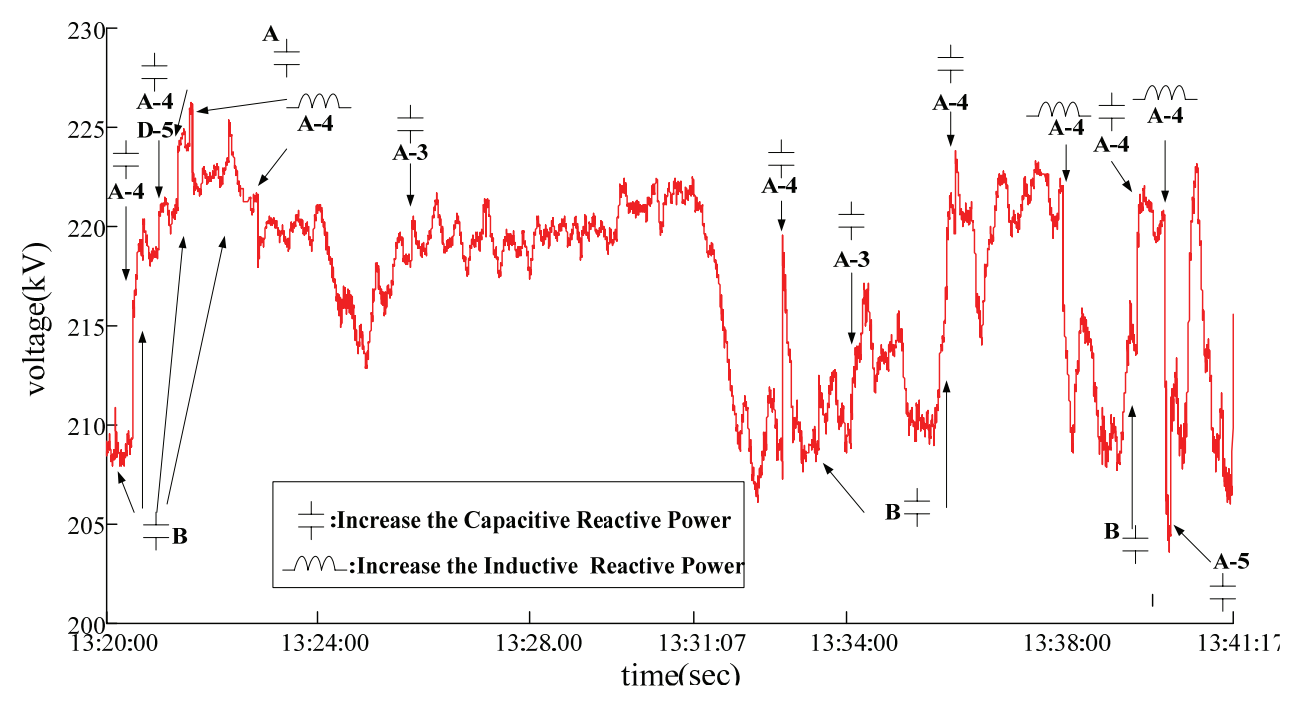

Fig. 4. The time sequence of reactive power compensation devices

Table 2. The step changes of $220 \mathrm{kV}$ buses of substations

\begin{tabular}{c|c|c}
\hline Gathering & \multicolumn{2}{|c}{$220 \mathrm{kV}$ Bus Voltage } \\
\cline { 2 - 3 } Substation & Before & After \\
\hline A & $208 \mathrm{kV}$ & $216 \mathrm{kV}$ \\
\hline B & $205 \mathrm{kV}$ & $209 \mathrm{kV}$ \\
\hline D & $214 \mathrm{kV}$ & $215 \mathrm{kV}$ \\
\hline
\end{tabular}

23.76Mvar in the A-2 wind farm was put into operation at $13: 41: 16.840$, resulting in step changes of the voltages of all $220 \mathrm{kV}$ gathering stations bus, as shown in Table 2 .

In the following $5.5 \mathrm{~s}$, the active load flow in $220 \mathrm{kV}$ transmission line almost kept constant (Fig. 5), whereas the system voltage gradually increased, up to $221 \mathrm{kV}$ at D,
$223 \mathrm{kV}$ at B, 235kV at A (Fig. 6), respectively.

Such slow dynamics of system voltage had never been observed in the similar faults [3] that happened in China before. The stage seemed unusual, because the system voltage should have been stable in milliseconds, but in fact, it kept increasing for seconds and finally was unstable.

After the investigation, the suspicion that the process was caused by the response of the SVCs had been excluded. All SVCs had reached their upper limits and worked as fixed capacitors, except those in A-1, A-7 and B-1. They acted in the constant voltage control mode, and had reduced their reactive production to depress the voltage as possible till they reached their limits about $5 \mathrm{~s}, 7 \mathrm{~s}$, and $10 \mathrm{~s}$ 


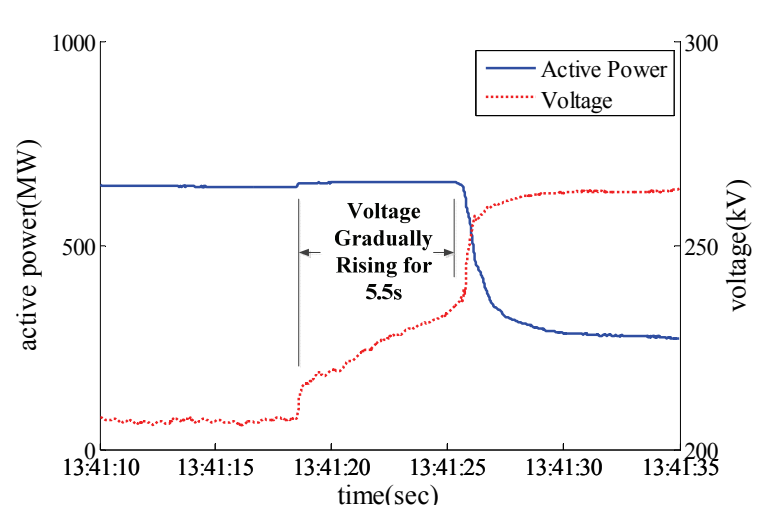

Fig. 5. The active power kept constant when the voltage increased gradually

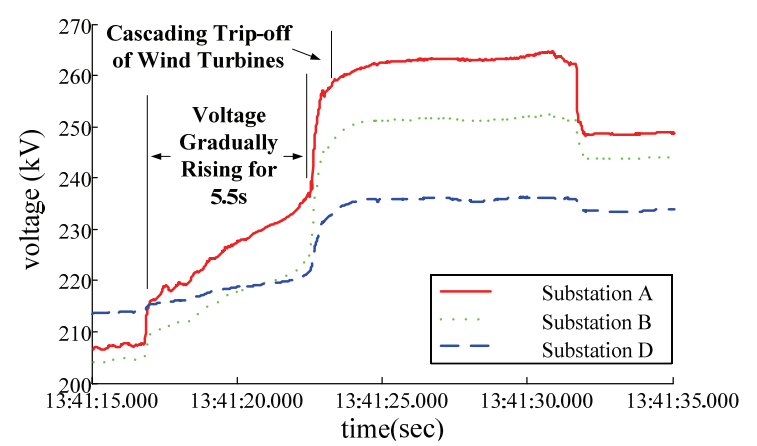

Fig. 6. The voltage increased slowly

later, respectively. Those actions of SVCs could not incur the system voltage changing in such a mode. The process could be neither caused by the reactive power from wind turbines, since the reactive output of wind turbines also kept zero.

The stage was, in fact, affected by the response of the active power of some DFIGs when the voltage changed. It would be explained in detail in Section 5.

\subsection{The stage of cascading}

At 13:41:22.220, the terminal voltage of A-3 and A-4 increased to more than $110 \%$ of their voltage rating, which was the threshold of overvoltage protection of wind turbines. Wind turbines of A-3 and A-4 tripped off at once. With the loss of the active power from the off-grid turbines, the reactive power became redundant, which caused the system voltage to increase to a higher level.

Like the domino, the cascading fault started. More and more wind turbines were forced to cut off due to overvoltage protection, and the system voltage grew higher and higher. The highest voltage was $263.173 \mathrm{kV}$ in the substation A, 251.496kV in B, and $236.069 \mathrm{kV}$ in $\mathrm{D}$, as shown in Figure 6. In 2s, 584 wind turbines of 15 wind farms tripped off with the total loss of $737.1 \mathrm{MW}$. Fig. 7 shows the time sequence of the wind turbine tripping (starting at 13:41:22.220).

After 8s later, reactive compensation devices quitted

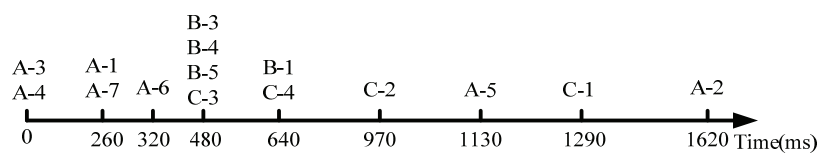

Fig. 7. The time sequence of wind turbine tripping (starting at $13: 41: 22.220$ )

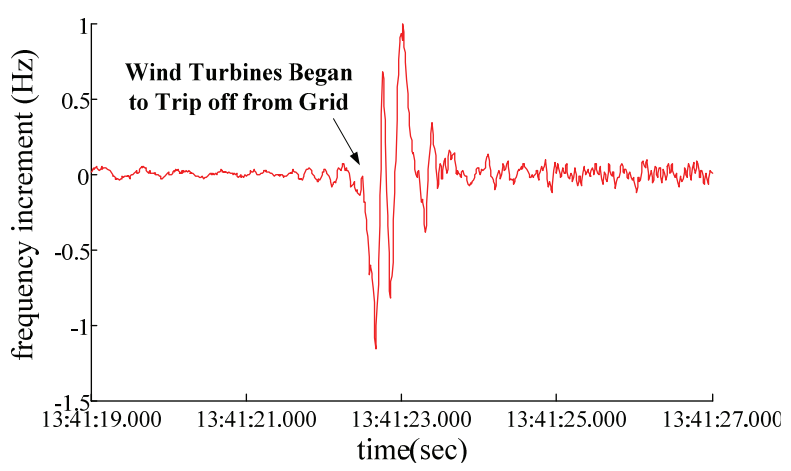

Fig. 8. The frequency fluctuations of the substation A

operation one by one due to their overvoltage protection, and recovered the system voltage to normal.

Due to the loss of a large amount of active power, the system frequency reduced. The lowest frequency in the $220 \mathrm{kV}$-level HV transmission system was $48.992 \mathrm{~Hz}$ (observed in the substation A), $49.149 \mathrm{~Hz}$ (in B), and the lowest frequency in the $500 \mathrm{kV}$-level EHV was $49.762 \mathrm{~Hz}$ (in D). The process of frequency fluctuations of the substation A is shown in Fig. 8. At the end of cascading, the system frequency was gradually restored to normal.

\section{Voltage Stability Analysis in Each Stage}

Based on the investigation, we can find out that this cascading fault expanded with the voltage changes, then incurred in system frequency fluctuation. The voltage change was the key factor to promote the fault evolution.

Many factors had impacts on the system voltage, including compensative devices, DFIGs and their protection settings and control strategies. They played different roles in each stage, and resulted in multi-time-scale dynamics of the voltage.

According to its time scale, voltage stability can be classified into 3 categories: static voltage stability, transient voltage stability and long-term voltage stability [13]. Therefore, it is essential in the fault analysis to identify of the nature of voltage stability in each stage.

\subsection{The Pre-event stage}

In this stage, the system ran near the extreme point of static voltage stability. Almost all capacitive compensation, including fixed capacitors and SVCs, had been put into use till the end of this stage. The system voltage fluctuated seriously, but it could still be corrected. The scene of the 
voltage profile shown in the PMU records could be reconstructed on the basis of the load flow model, using the P-U and Q-U sensitivity analysis method. See the detailed analysis in Section 5.1.

\subsection{The critical stage before cascading}

It is a little difficult to identify the category of voltage instability in this stage. At first, the system voltage increased simultaneously once the $23.76 \mathrm{MW}$ capacitor group was switched on, and then kept increasing for 5.5 seconds. Such dynamics of the system voltage was unusual, because its mechanism could not be explained by the conventional theory of voltage stability: First, it was almost impossible incurred by static voltage instability, because it has been calculated that the system still had margins to the extreme point of the PV curve (the well-known "nose curve") before the cascading fault.

Second, it could be hardly classified into transient voltage instability. Recently, dynamic bifurcation theory has been widely used in analyzing transient voltage stability in the power system with DFIGs [4]. Types of bifurcation have been revealed in numerical simulation, including Limit Induced Bifurcation (LIB), Singular Induced Bifurcation (SIB), Period bifurcation (PB). However, bifurcation theory was hardly helpful, because there were some doubts: (1) Time scale and the voltage profile. Once LIB and SIB happens, the voltage almost collapses instantaneously, whereas the voltage in this case kept increasing for seconds before out of control. (2)The generator portfolio: The power system that is threatened by LIB or SIB always consists of synchronous generators or motors, whereas the system in this case was composed of nearly pure wind turbines.

Finally, the fault was also impossible incurred by the long-term voltage stability, because the typical duration range of long-term voltage stability is for a few minutes, typically for $2-3 \mathrm{~min}$ or longer. The possibility was also excluded after the investigation, because none of equipment (such as heat load, under-load tap changer) that could response in the same time scale had taken action.

It is deduced that this stage did not fall in any known type of voltage instability. It probably belongs to midterm voltage dynamics. DFIGs and SVCs should have participated in the system dynamics. See the detailed analysis in Section 5.2.

\subsection{The stage of cascading trip-off of wind turbines}

Since the voltages had reached the threshold of overvoltage protection of wind turbines, some wind turbines tripped off and the wind power through transmission lines decreases significantly. At the same time, the reactive power compensation devices still kept working because of their longer protection delay and higher protection threshold, which resulted in more wind turbine trip-off due to the overvoltage protection in the repeated mode. This process is known as the common mode tripping of wind turbines [14].

Previous studies have provided a thorough inventory of the theory analysis and simulation verification of the common mode tripping of turbines. This paper no longer makes a detailed analysis.

\section{Simulation Models and Reproduction Studies}

According to the actual data, we have reconstructed the scene that day using the MATLAB Power System Analysis Toolbox (PSAT) [15]. Based on the PMU recording data, the pre-event power flow conditions are elaborately adjusted to match the actual situations.

\subsection{Reproduction study on voltage fluctuations in the Pre-event stage and the cascading stage}

The voltage fluctuation in these two stages can be simulated based on power flow models. Some remarks:

(1) DFIGs are taken as the negative constant PQ load;

(2) SVCs that work in the constant voltage mode are taken as the PV nodes with Q limits; SVCs that work in the constant $\mathrm{Q}$ mode are taken as the negative constant PQ load; SVCs that have reached their limits are taken as the constant shunt capacity branches.

(3) The $500 \mathrm{kV}$ bus of the substation D is strong enough, so it is taken as the slack bus.

Suppose that there are $N$ nodes in the system, including 1 slack bus, $r$ PV nodes and $n-r$ PQ nodes $(n=N-1)$. The linearization of the power flow equation is as follows:

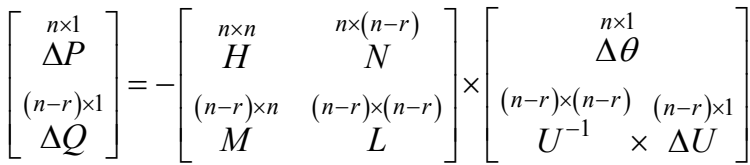

where $\Delta P$ and $\Delta Q$ denote the increment of active and reactive power, respectively; $\Delta \theta$ is the increment of voltage phase angle; $\Delta U$ denotes the increment of voltage amplitude; $U$ is the node voltage; $H, N, M, L$ denote the four sub-block matrixes of the Jacobian matrix.

In a particular operating point, let $\Delta P=0$ :

$$
\begin{aligned}
0 & =-H \Delta \theta-N U^{-1} \Delta U \\
\Delta Q & =-M \Delta \theta-L U^{-1} \Delta U
\end{aligned}
$$

Eliminate $\Delta \theta$ in Eq. (2):

$$
U^{-1} \Delta U=\left(M H^{-1} N-L\right)^{-1} \Delta Q=S_{u Q} \Delta Q
$$


Where $S_{u Q}$ is the Q-U sensitivity matrix whose element denotes the voltage change $\Delta U$ with respect to the injection change of the reactive power $\Delta Q$.

The Q-U sensitivity can be used to analyze the voltage fluctuation caused by reactive device operations in Section 3.1. For example, as the reactive power in any wind farm that is connected with the substation A increases or decreases 1Mvar, the simulated voltage changes in the $220 \mathrm{kV}$ bus are ranged from $5.3-5.5 \mathrm{kV}$, which matches the PMU recorded data well. The similar verification can be done in other wind farms.

Similarly, assume $\Delta Q=0$ :

$$
\begin{aligned}
\Delta P & =-H \Delta \theta-N U^{-1} \Delta U \\
0 & =-M \Delta \theta-L U^{-1} \Delta U
\end{aligned}
$$

Eliminate $\Delta \theta$ in Eq. (4):

$$
U^{-1} \Delta U=\left(-M H^{-1} N+L\right)^{-1} \times M H^{-1} \Delta P=S_{u p} \Delta P
$$

Here, $S_{u p}$ is the sensitivity matrix whose element denotes the voltage change $\Delta U$ with respect to the injection change of active power $\Delta P$.

The P-U sensitivity can be used to analyze the voltage fluctuation caused by the active power loss from wind turbines in Section 3.3. For example, as the active power output of any wind farm connected to the substation A decreases $1 \mathrm{MW}$, the simulated voltage changes in the $220 \mathrm{kV}$ bus are ranged from $4.7 \mathrm{kV}-5.7 \mathrm{kV}$, which matches the PMU recorded data well. So does other wind farms.

\subsection{Reproduction study on Mid-term voltage dynamics in the critical stage}

The voltage dynamics in this stage was affected by the slow dynamics of some DFIGs. This subsection will reveal how the conclusion is drawn.

The DIFGs are handled in two ways:

(1) Some are taken as the negative constant PQ, because the PMU data had shown that those DFIGs kept constant when the voltage changed, identical with the ideal constant PQ model. The DFIGs of this type was up to $53.1 \%$.

(2) Other must be modeled with dynamic response. The PMU records had shown that the response of their active power was sensitive to the voltage, whereas the reactive power almost kept unchanged. The active power increased simultaneously when there was a step increment in the voltage, and then gradually restored to the original value (as shown in Fig. 9). The DFIGs of this type was up to $46.9 \%$.

First, we use the full order model [16] to simulate the DFIGs of the second type. Based on the stator-fluxoriented vector control method, the DFIG rotor-side

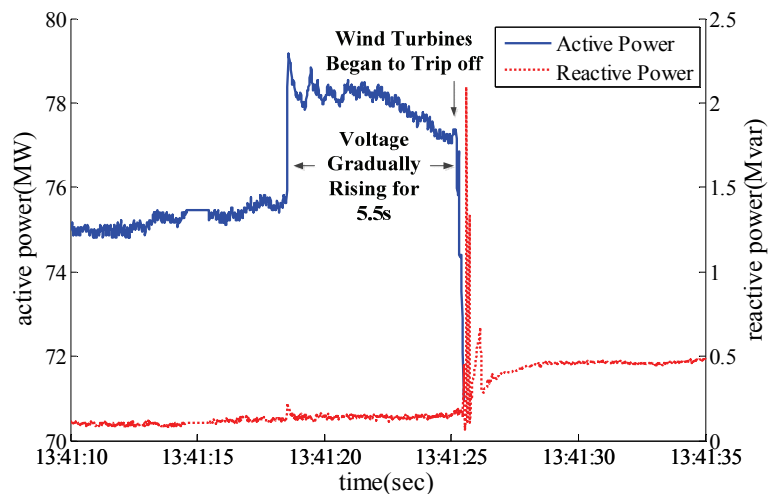

Fig. 9. The response of the active and reactive power of wind turbines to the voltage changes

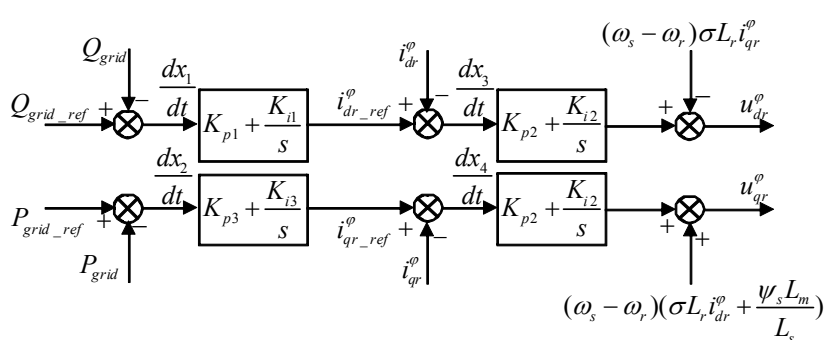

Fig. 10. The control model of the DFIG rotor-side converter

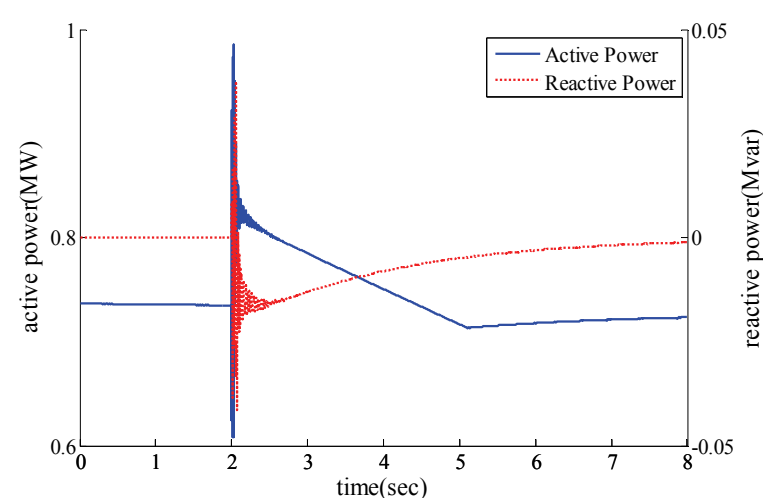

Fig. 11. The responses to the step voltage increment based on the full order model

converter applies decoupled control to the active and reactive power, which is achieved by regulating the rotor current to control the rotor voltage. The response of the active power is decided by the proportional gain $\left(K_{p 2}\right)$ and integration gain $\left(K_{i 2}\right)$ of DFIG rotor-side converter [17]. The control model of the DFIG rotor-side converter is shown as Fig. 10.

Using the full-order model, we can simulate the response of the active and reactive power of a DFIG with respect to the step voltage increment, and their profiles are verified to match the PMU records well, as shown in Fig. 11.

Although the dynamics of DFIGs could be reproduced well using the full-order model, it was almost impossible to reconstruct the scene with it, because there were 1191 wind turbines of 23 wind farms in the system; it was impossible to collect all control strategies and their parameters of 
various wind turbines, since they were protected by some commercial secrets. According to the measured response of wind turbines, an equivalent dynamic load model is recommended in this paper [18]. The mathematical model is as follows:

$$
\begin{gathered}
\dot{x}_{P}=-x_{P} / T_{P}+P_{s}-P_{t} \\
P=x_{P} / T_{P}+P_{t} \\
P_{s}=P_{0}\left(V / V_{0}\right)^{\alpha_{s}} \\
P_{t}=P_{0}\left(V / V_{0}\right)^{\alpha_{t}}
\end{gathered}
$$

Where $P$ denotes the active power variable of the DFIG, $P_{s}$ denotes the static component, and $P_{t}$ denotes the transient component, and $P_{0}$ denotes the steady value; $V$ denotes the voltage of the DFIG stator, and $V_{0}$ denotes the steady value, too. The active power of the DFIG is sensitive to the stator voltage, so $\alpha_{s}$ and $\alpha_{t}$ denote static exponent and transient exponent; $T_{P}$ represents the time constant. $x_{P}$ is defined as a middle variable whose dimension is energy.

Again, using the equivalent model, we simulate the response of the active and reactive power of a single DFIG, shown in Fig. 12. Except the high frequency component that decays quickly, the profile matches in general.

The mathematical model of the SVC is as follows [15]:

$$
\dot{b}_{S V C}=\left(K_{r}\left(V_{r e f}-V\right)-b_{S V C}\right) / T_{r}
$$

where $T_{r}$ is the time constant, $K_{r}$ denotes the magnification of the SVC, $V_{r e f}$ and $V$ are reference voltage and compensation point voltage, and $b_{S V C}$ is the equivalent susceptance of the SVC.

The reactive power of the $\mathrm{SVC}$ is:

$$
Q=b_{S V C} V^{2}
$$

The scene is simulated by two means: Case $A$ uses the negative constant PQ loads to model all wind turbines; Case $B$ uses the proposed model.

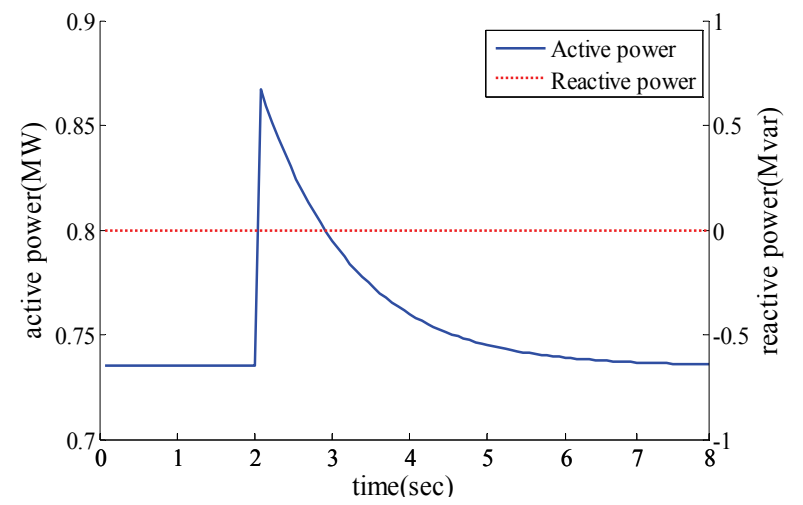

Fig. 12. The response to the step voltage increment based on the proposed model

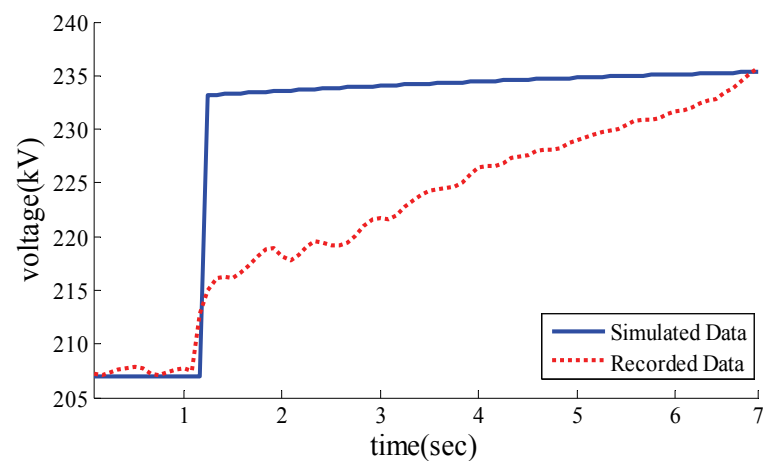

Fig. 13. The reproduction of the voltage profile of the substation A (Case A: Constant PQ model)

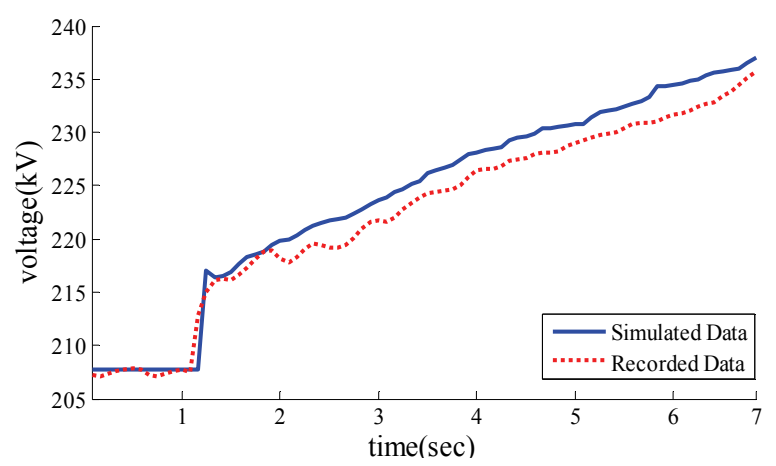

Fig. 14. The reproduction of the voltage profile of the substation A (Case B: the proposed model)

In Case A, Fig. 13 compares records and simulated data (the $220 \mathrm{kV}$ bus voltage of the substation A). Obviously, the voltage profile does not match in the following aspects:

(1) The initial value of the voltage change $\left(t_{0+}\right)$. The simulated value is much larger than that in the record.

(2) The dynamic response of the voltage. Only a stepresponse-like process can be found in the figure.

We also simulate the same scene using the proposed equivalent dynamic load model. The comparison between the records and simulated curve is shown in Fig. 14. Two curves match much better. We can conclude that the slow response of the active power of some DFIGs had contributed to the slow increasing of the system voltage. In general, it was not the dangerous factor of voltage instability. On the contrary, it was helpful to prevent the system voltage from increasing too quickly.

\section{Conclusion}

Based on PMU records, this paper has investigated the entire process of the cascading trip-off fault in North China on May 14, 2012, and analyzed the mechanism of the voltage instability through the scene reproduction. The conclusion is summarized as follows: 
(1) The severe fault could be divided into three stages: the pre-event stage, the critical stage before cascading, and the cascading stage. The voltage change was the key to affect the evolution of the fault.

(2) The voltage fluctuation in the pre-event stage was mainly caused by the operation of reactive compensation devices and wind speed variation; the slow increasing of the voltage in the critical stage was affected by the dynamics of the active power of some DFIGs; the overvoltage was enhanced by the loss of wind turbines, and then conversely higher overvoltage resulted in more tripping of turbines.

(3) In the reconstruction, most of DFIGs can be taken as the negative constant PQ load, whereas some DFIGs should be handled with nonlinear dynamic characteristics. This paper has proposed the equivalent voltage sensitive dynamic model for fast computation. Simulation has validated the model. In this case, DFIGs were helpful in voltage stability.

The large-scale wind turbine cascading trip-off accident happens sometime, which is closely related to the factors such as the grid structure, wind turbine operation modes, and control strategies of reactive compensation devices. The dynamic process of the fault could be quite complex, and more work must be done in modeling the actual system.

\section{Acknowledgements}

This work was supported by Project No: 2013 BAA01B03 and SGJB0000TKJS1500017.

\section{References}

[1] World Wind Energy Association (WWEA), World Wind Energy Half-year Report 2014, September. 2014.

[2] Union for the Coordination of Transmission of Electricity (UCTE), Final Report System Disturbance on 4 November 2006, Brussels, Belgium: UCTE, 2006.

[3] D. Xu, W. Hu, F. Gao and X. H. Yang," Analysis on Mechanism and Preventing Strategies of Cascading Tripping out of Numerous Wind Turbines in China," in 2014 International Conference on Power System Technology, Chengdu, China, Oct. 2014.

[4] K. B. Kilani, M. Elleuch. "Structural Analysis of Voltage Stability in Power Systems Integrating Wind Power," IEEE Trans. Power System, vol.28, no.4, pp.3785-3794, Nov. 2013.

[5] S. K. Konar, D. Chatterjee, and S. Patra, "VQsensitivity-based index for assessment of dynamic voltage stability of power systems, "IET Generation, Transmission \& Distribution, vol.9, no.7, pp.677-685,
April. 2015.

[6] Y. Z. Lin, L. B. Shi, L. Z. Yao, Y. X. Ni, S. Y. Qin, R. M. Wang, J. P. Zhang. "An analytical solution for voltage stability studies incorporating wind power", Journal of Electrical Engineering \& Technology, vol.10, no.3, pp.865-876, May, 2015

[7] V. S. S. Kumar, K. K. Reddy, and D. Thukaram, "Coordination of Reactive Power in Grid-Connected Wind Farms for Voltage Stability Enhancement," IEEE Trans. Power System, vol.29, no.5, pp.23812390, Sep.2014.

[8] N. R. Ullah, and T. Thiringer, "Variable Speed Wind Turbines for Power System Stability Enhancement," IEEE Trans. Energy Conversion, vol.22, no.1, pp.5260, Mar. 2007

[9] E.H. Kim, J.H. Kim, S.H. Kim, J. Choi, K.Y. Lee, H.C. Kim, "Impact Analysis of Wind Farms in the Jeju Island Power System," IEEE Systems Journal, vol. 6, no. 1, pp. 134-139, Mar. 2012

[10] X. Ye, Y. Qiao and Z.X. Lu, "Cascading Tripping out of Numerous Wind Turbines in China: Fault Evolution Analysis and Simulation Study," in Power and Energy Society General Meeting, San Diego, Canada, July 2012.

[11] V. Akhmatov and P. B. Eriksen, "A Large Wind Power System in Almost Island Operation - A Danish Case Study", IEEE Trans. Power System, vol. 22, no. 3, pp. 937-943, Aug. 2007

[12] J. Lu, Y. Qiao, Z. X Lu and J.Y. Ruan, "Survey and Study on the Overvoltage Fault Evolution of A Largescale Wind Power Base," in International Conference on Wind energy Gird-Adaptive Technologies, Jeju, Korea, Oct. 2014.

[13] C.W. Taylor. Power System Voltage Stability. New York: McGraw-Hill, Companies, Inc, 1994:12-15.

[14] Richard Piwko, Nicholas Miller, and Juan SanchezGasca, et al. "Integrating Large Wind Farms into Weak Power Grids with Long Transmission Line," in Power Electronics and Motion Control Conference, Shanghai, China, August 2006.

[15] F. Milano, Power System Analysis Toolbox: Documentation for PSAT version 1.3.4, Nov. 2002.

[16] G. Byeon, I. Park, and G. Jang, "Modeling and control of a doubly-fed induction generator (DFIG) wind power generation system for real-time simulations", Journal of Electrical Engineering \& Technology, vol. 5, no. 1, pp. 61-69, Mar. 2010

[17] F. Wu, X. P. Zhang, K. Godfrey, P. Ju. "Small Signal Stability Analysis and Optimal Control of a Wind Turbine with Doubly Fed Induction Generator," IET Generation, Transmission \& Distribution, vol.1, no.5, pp.751-760, Aug. 2007.

[18] D. Karisson and D. J. Hill, "Modeling and Identification of Nonlinear Dynamic Loads in Power Systems," IEEE Trans. Power Systems, vol.9, no.1, pp.157-166, Feb.1994. 


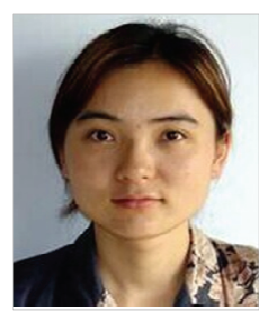

Ying Qiao She received the B.S. and $\mathrm{Ph} . \mathrm{D}$. degrees in electrical engineering from Shanghai Jiaotong University, Shanghai, and Tsinghua University, Beijing, China, in 2002 and 2008, respectively. She is now a lecturer of Electrical Engineering at Tsinghua University, Beijing, China, where she has been employed since 2010. Her research interests include renewable energy and power system security and control.

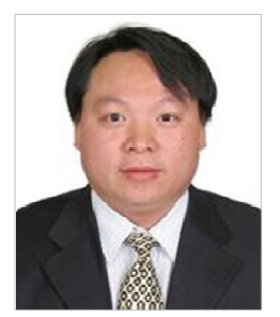

Zong-Xiang Lu He received the B.S. and Ph.D. degrees in electrical engineering from Tsinghua University, Beijing, China, in 1998 and 2002, respectively. $\mathrm{He}$ is now an associate Professor of Electrical Engineering at Tsinghua University, Beijing, China, where he has been employed since 2002. His research interests include power system reliability, renewable energy and micro grid, and large scale wind power integration.

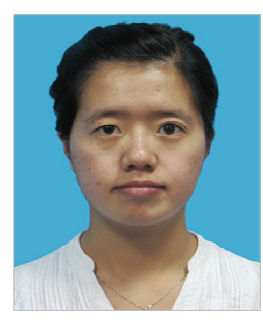

Ji Lu She received B.S. degree in electrical engineering from College of Science and Technology of China Three Gorges University, Yichang, China, in 2012. She is a postgraduate in China Three Gorges University, Yichang, China. Currently, she is studying at Tsinghua University, Beijing, China. Her research interests include reactive power control and renewable energy.

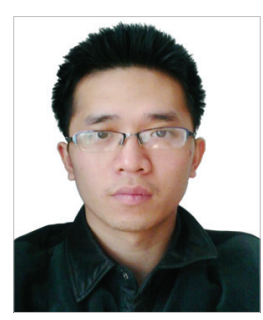

Jia-Yang Ruan He received B.S. and M.S. degrees in electrical engineering from Tsinghua University, Beijing, China, in 2010 and 2012, respectively. His research work concerns stability analysis of grid-connected wind turbines, and application of aggregated modeling for wind farms.

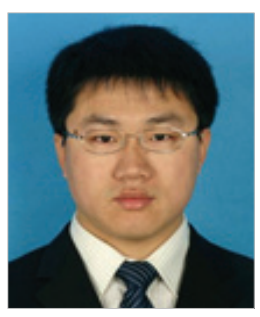

Lin-lin Wu He received the B.S. and M.S. degrees in electrical engineering from Tsinghua University, Beijing, China, in 2008 and 2010, respectively. $\mathrm{He}$ is now an engineer of smart grid and renewable resource technology department at North China Electric Power Research Institute. His research interests include large scale wind power integration, power grid dispatch and operation. 